\title{
Donor Heart Utilization following Cardiopulmonary Arrest and Resuscitation: Influence of Donor Characteristics and Wait Times in Transplant Regions
}

\author{
Mohammed Quader, Luke Wolfe, Gundars Katlaps, and Vigneshwar Kasirajan \\ Department of Cardiothoracic Surgery, Virginia Commonwealth University, Richmond, VA 23298, USA \\ Correspondence should be addressed to Mohammed Quader; mquader@mcvh-vcu.edu
}

Received 5 May 2014; Accepted 20 June 2014; Published 8 July 2014

Academic Editor: Parmjeet Randhawa

Copyright (C) 2014 Mohammed Quader et al. This is an open access article distributed under the Creative Commons Attribution License, which permits unrestricted use, distribution, and reproduction in any medium, provided the original work is properly cited.

Background. Procurement of hearts from cardiopulmonary arrest and resuscitated (CPR) donors for transplantation is suboptimal. We studied the influences of donor factors and regional wait times on CPR donor heart utilization. Methods. From UNOS database (1998 to 2012), we identified 44,744 heart donors, of which 4,964 (11\%) received CPR. Based on procurement of heart for transplantation, CPR donors were divided into hearts procured (HP) and hearts not procured (HNP) groups. Logistic regression analysis was used to identify predictors of heart procurement. Results. Of the 4,964 CPR donors, 1,427 (28.8\%) were in the HP group. Donor characteristics that favored heart procurement include younger age $(25.5 \pm 15$ yrs versus $39 \pm 18$ yrs, $P \leq 0.0001)$, male gender ( $34 \%$ versus $23 \%, P \leq 0.0001)$, shorter CPR duration $(<15 \mathrm{~min}$ versus $>30 \mathrm{~min}, P \leq 0.0001)$, and head trauma $(60 \%$ versus 15\%). Among the 11 UNOS regions, the highest procurement was in Region 1 (37\%) and the lowest in Region 3 (24\%). Regional transplant volumes and median waiting times did not influence heart procurement rates. Conclusions. Only $28.8 \%$ of CPR donor hearts were procured for transplantation. Factors favoring heart procurement include younger age, male gender, short CPR duration, and traumatic head injury. Heart procurement varied by region but not by transplant volumes or wait times.

\section{Introduction}

For patients with advanced heart failure awaiting heart transplantation (HTx), donor heart supply remains a limiting factor in offering the ultimate treatment option. Efforts to optimize management of potential heart donors have led to increased utilization of donor hearts [1], yet this increase falls far short of the existing demands on organs for transplantation [2]. New avenues that would increase available donor hearts have been explored, including donation after cardiac death [3], ex vivo organ resuscitation [4], and, importantly, extended donor selection criteria [5]. Of these extended criteria, cardiopulmonary arrest and resuscitated (CPR) organ donors have significantly increased the potential organ donor pool. In the past decade alone, there has been a $90 \%$ increase in the number of organ donors who were successfully resuscitated after cardiopulmonary arrest [2] (Figure 1). We previously reported that the clinical outcomes of heart transplantation from CPR donors are similar to the outcomes from non-CPR donors [6]. This finding was also noted in other solid organ transplantation studies $[7,8]$.

Despite these encouraging reports, utilization of CPR donor hearts has been less than 30\% [2]. The reasons are multifactorial, including concerns regarding warm ischemic damage to organs sustained during cardiopulmonary arrest [9], nonuniform donor selection criteria among transplantation centers, acuity of illness of the organ recipients, and, perhaps, the overall transplant volumes and experiences. Most published studies on this subject have focused on the recipient outcomes with donor variables studied primarily to predict posttransplantation outcomes $[10,11]$. Our study principally focuses on the donor variables, with the aim of identifying CPR donor characteristics predictive of heart procurement for transplantation. We also sought to study the influences of 11 UNOS regional heart transplantation volumes and regional wait times on CPR donor heart utilization. 


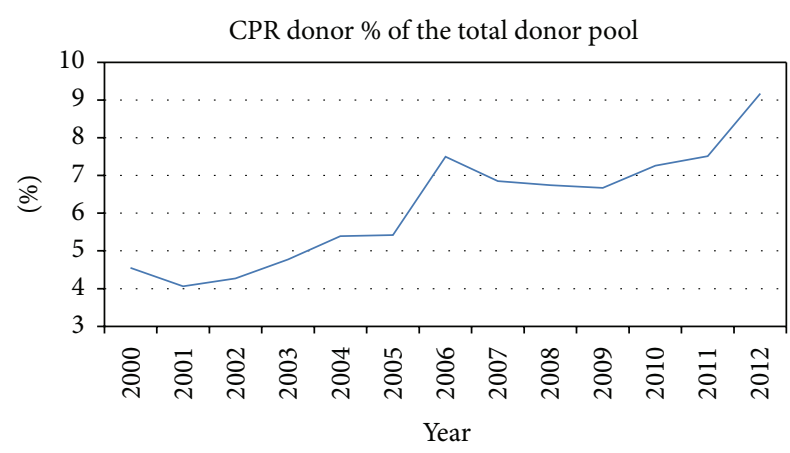

FIGURE 1: CPR donor percentage of the total heart donors.

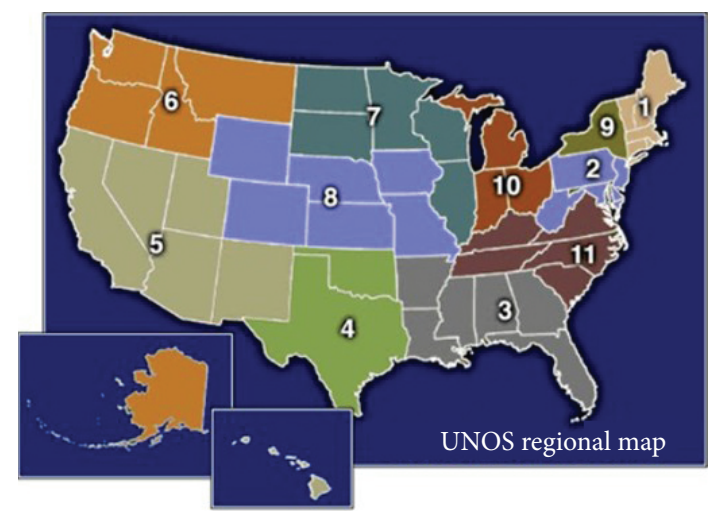

FIGURE 2: UNOS 11 heart transplantation regions in USA.

\section{Material and Methods}

We examined the UNOS heart donor data between 1998 and 2012 and identified 44,744 consented heart donors, of which 4,964 (11\%) were from CPR donors. This comprised our study group, which was further divided into two groups based on heart procured (HP) or heart not procured (HNP) for transplantation. Besides donor demographics, data on comorbid conditions, social history, causes of death, and duration of CPR were collected in a deidentified fashion for analysis (Table 1). Data on 11 UNOS transplantation regions (Figure 2) were gathered and analyzed for transplantation volumes, median $1 \mathrm{~A}$ status wait times for heart transplantation, and percent CPR donor heart utilization (Table 2).

Numeric data were analyzed and reported as mean, median, and standard deviations. A $P$ value of less than 0.05 is considered statistically significant. Continuous variables were analyzed with Student's $t$-test; categorical variables were analyzed with the chi-square test. A logistic regression analysis of 11 donor variables was performed to identify predictors of heart procurement for transplantation from the CPR donor pool. The results of this regression analysis are reported as odds ratios with confidence limits.

\section{Results}

During the study period of 1998 to 2012, a total of 44,744 consented heart donors were identified in the UNOS database.
TABLE 1: CPR donor characteristics.

\begin{tabular}{|c|c|c|c|}
\hline \multirow[b]{2}{*}{ Donor variable } & \multicolumn{3}{|c|}{ Heart procured for transplantation } \\
\hline & $\begin{array}{c}\text { Yes } \\
N=1,427 \\
(28.8 \%)\end{array}$ & $\begin{array}{c}\text { No } \\
N=3,537 \\
(67.2 \%)\end{array}$ & $P$ value \\
\hline Age in years & $25.5 \pm 15$ yrs & $39 \pm 18$ yrs & $<0.0001$ \\
\hline Gender & & & $<0.0001$ \\
\hline Male & 34 & 66 & \\
\hline Female & 23 & 77 & \\
\hline Duration of CPR & & & $<0.0001$ \\
\hline$<15 \min$ & 31 & 69 & \\
\hline $15-30 \mathrm{~min}$ & 29 & 71 & \\
\hline$>30 \mathrm{~min}$ & 25 & 75 & \\
\hline Cause of Death & & & $<0.0001$ \\
\hline Head trauma & 60 & 40 & \\
\hline Anoxia & 29 & 71 & \\
\hline $\begin{array}{l}\text { Cerebrovascular/ } \\
\text { stroke }\end{array}$ & 15 & 85 & \\
\hline Ethnicity & & & $<0.0001$ \\
\hline Hispanic & 37 & 63 & \\
\hline Black & 33 & 67 & \\
\hline White & 27 & 73 & \\
\hline Asian & 24 & 76 & \\
\hline Donor $\mathrm{ABO}$ & & & $<0.0001$ \\
\hline $\mathrm{O}$ & 32 & 68 & \\
\hline $\mathrm{A}$ & 27 & 73 & \\
\hline B & 24 & 76 & \\
\hline $\mathrm{AB}$ & 15 & 85 & \\
\hline Hypertension & & & $<0.0001$ \\
\hline Yes & 12 & 88 & \\
\hline No & 35 & 65 & \\
\hline
\end{tabular}

Social history

Cigarette use

$<0.0001$

$\begin{array}{lll}\text { Yes } & 17 & 83\end{array}$

$\begin{array}{lll}\text { No } & 34 & 66\end{array}$

Heavy ETOH use

$\begin{array}{lll}\text { Yes } & 22 & 78 \\ \text { No } & 30 & 70\end{array}$

\begin{tabular}{llll}
\hline UNOS region & & & $<0.0075$ \\
1 & 37 & 63 & \\
3 & 24 & 76 & \\
\hline
\end{tabular}

3 or more inotropic

agents at the time of

$<0.0001$

incision

\begin{tabular}{cccc} 
Yes & 12 & 88 & \\
No & 30 & 70 & \\
\hline $\begin{array}{l}\text { LV ejection fraction } \\
\%\end{array}$ & $61.4 \pm 9.0$ & $48.2 \pm 17.3$ & $<0.0001$
\end{tabular}

CPR: cardiopulmonary resuscitation; ABO: blood groups; ETOH: alcohol; UNOS: United Network of Organ Donation; LV EF: left ventricle ejection fraction.

Of which, 4,964 (11\%) donors sustained cardiopulmonary arrest and were resuscitated to spontaneous rhythm and 
TABLE 2: Heart transplantation volumes, median wait times, and CPR donor heart procured for transplantation by 11 UNOS regions.

\begin{tabular}{lccccc}
\hline Region & Total population & Population/HTx & Mean HTx volume/yr & Median 1A wait time in days & CPR donor utilization-\% \\
\hline 1 & $13,936,692$ & 158,371 & 88 & 59.6 & 37 \\
2 & $30,917,426$ & 110,026 & 281 & 40.3 & 29 \\
3 & $48,262,570$ & 165,851 & 291 & 47.6 & 24 \\
4 & $29,874,023$ & 140915 & 212 & 34.6 & 32.6 \\
5 & $52,294,441$ & 155,176 & 337 & 90.3 & 26 \\
6 & $15,521,147$ & 242,517 & 64 & 80.3 & 30 \\
7 & $25,513,744$ & 125,683 & 203 & 58.3 & 30 \\
8 & $19,601,598$ & 141,018 & 139 & 68.6 & 29 \\
9 & $20,196,272$ & 133,750 & 151 & 67.6 & 28 \\
10 & $27,974,919$ & 136,463 & 205 & 239 & 31 \\
11 & $33,498,321$ & 140,160 & & & \\
\hline
\end{tabular}

HTx: heart transplantation; CPR: cardiopulmonary resuscitation.

circulation. Only 1,427 (28.8\%) of these CPR donor hearts were procured for transplantation; these donors comprised the hearts procured (HP) group.

When compared to the hearts not procured group, the hearts procured group were relatively younger $(25 \pm 15$ years versus $39 \pm 18$ years, $P \leq 0.0001)$ and a high proportion of them were male gender $(34 \% \mathrm{M}$, versus $23 \% \mathrm{~F}, P \leq$ 0.0001 ). Cause of death influenced the percent utilization of CPR donor hearts: those with head trauma leading to brain death were selected more often for heart procurement compared to those with intracerebral bleed $(60 \%$ and $15 \%$, resp., $P \leq 0.0001)$. Similarly, mechanism of death played a role in the selection of CPR donors for heart procurement: those with gunshot wounds (44\%), asphyxiation (40\%), or blunt trauma (39\%) were more often selected for heart procurement compared to those with stroke (15\%). CPR donors with a history of hypertension were selected less often for heart procurement, as only $12 \%$ of the hearts procured group had a history of hypertension, compared to $35 \%$ in the hearts not procured group $(P \leq 0.0001)$. Ethnicity and the social history of the CPR donor were also factored into the heart procurement for transplantation (Table 1). Notably, a higher percentage of donors with Hispanic ethnicity (37\%) were accepted for heart procurement compared to only $24 \%$ for Asian ethnicity $(P \leq 0.0001)$. We noticed that there was a higher prevalence of head trauma (35\%), male gender (62\%), and blood group O (60\%) in Hispanic ethnicity heart donors compared to other heart donors. Donors with a history of tobacco abuse (17\%) and heavy alcohol consumption (22\%) were less often selected for heart procurement. Duration of CPR also contributed significantly to the selection of donor heart for transplantation. Those donors with a CPR duration of less than $15 \mathrm{~min}$ were selected more often for heart procurement than those with a CPR duration of greater than $30 \mathrm{~min}$ ( $31 \%$ and $25 \%$, resp., $P \leq 0.0001)$. Left ventricle ejection fraction (EF) was near normal in heart procured group compared to heart not procured group, $61.4 \pm 9.0$ versus $48.2 \pm 17.3, P<0.0001$.

Data on regional heart transplantation volumes, wait times on the transplantation list, and CPR donor heart utilization were also studied (Table 2). Heart transplantation volumes, measured as the mean number of transplantations performed per year, varied between the 11 UNOS regions, from 64 in Region 6 to 337 in Region 5. Median wait times on status $1 \mathrm{~A}$ listing also varied significantly between the regions and did not correlate with transplantation volumes. The longest median wait time was noted in Region 7 (90 days) while the shortest was in Region 5 (35 days).

CPR donor heart utilization varied significantly between the eleven regions, with a low of $24 \%$ in Region 3 to a high of $37 \%$ in Region $1(P \leq 0.0001)$. We also evaluated the CPR donor utilization by population per heart transplantation performed in the 11 regions. Region 6 had the highest population per heart transplant $(242,517 / \mathrm{HTx})$, while the lowest was in Region $2(110,026 / \mathrm{HTx})$. CPR donor utilizations in Regions 6 and 2 were $26 \%$ and $29 \%$, not statistically significant.

Logistic regression analysis using heart procurement for transplantation from CPR donors as an outcome variable was studied factoring in eleven donor variables, including regional transplant volumes, and wait times on transplantation list (Table 3). CPR donor characteristics associated with heart procurement for transplantation by logistic regression analysis include younger donor age, male gender, shorter CPR duration, head trauma as a cause of death versus cerebrovascular accident, Hispanic ethnicity, blood group $\mathrm{O}$, and location in Region 1 . Factors that were negatively associated with heart procurement for transplantation were need for multiple inotropes at the time of organ procurement, history of substance abuse, nontraumatic injuries leading to brain death, low EF, and location in Region 3 (Table 3).

\section{Comment}

Since the limiting factor in offering heart transplantation to patients with advanced heart failure is a suitable heart donor, we embarked on studying a group of underutilized potential heart donors: those who sustained cardiopulmonary arrest and were resuscitated. Our study demonstrates that CPR donors that are younger, are male, incurred head trauma, received shorter duration $\mathrm{CPR}$, and did not smoke or abuse alcohol are selected more often for heart procurement. We did 
TABLE 3: CPR donor variables that are predictive of successful heart procurement for transplantation by logistic regression analysis.

\begin{tabular}{lccc}
\hline Donor variable & Odds ratio & $\begin{array}{c}95 \% \text { confidence } \\
\text { limits }\end{array}$ & $P$ value \\
\hline Age in years & 1.033 & $1.028-1.038$ & $<0.0001$ \\
\hline $\begin{array}{l}\text { Gender } \\
\quad \text { Female versus male }\end{array}$ & 1.470 & $1.272-1.699$ & $<0.0001$ \\
\hline $\begin{array}{l}\text { Comorbidities } \\
\quad \text { Hypertension: yes } \\
\text { versus no }\end{array}$ & 1.981 & $1.591-2.467$ & $<0.0001$ \\
\hline $\begin{array}{l}\text { Duration of CPR } \\
\begin{array}{l}\text { Cause of death versus } \\
\text { head trauma }\end{array}\end{array}$ & 1.013 & $1.008-1.017$ & $<0.0001$ \\
$\quad \begin{array}{l}\text { Anoxia } \\
\text { Cerebrovascular/ } \\
\text { stroke }\end{array}$ & 1.118 & $0.945-1.322$ & 0.2190 \\
\hline
\end{tabular}

Ethnicity versus

Hispanic

$\begin{array}{llll}\text { White } & 1.261 & 1.012-1.570 & 0.5255 \\ \text { Black } & 0.939 & 0.725-1.215 & 0.2575 \\ \text { Asian } & 1.125 & 0.626-2.020 & 0.9644\end{array}$

\begin{tabular}{lccc}
\hline ABO versus $O$ & & & \\
$\mathrm{~A}$ & 1.181 & $1.014-1.376$ & 0.0002 \\
$\mathrm{AB}$ & 3.278 & $2.071-5.189$ & $<0.0001$ \\
$\mathrm{~B}$ & 1.617 & $1.287-2.032$ & 0.8244 \\
\hline
\end{tabular}

Social history

Cigarette use: yes

versus no

$1.288 \quad 1.062-1.563$

0.0103

History of cocaine use

1.981

$1.591-2.467$

0.3330

\begin{tabular}{cccc}
\hline UNOS region versus 1 & & & \\
2 & 1.634 & $0.990-2.696$ & 0.1654 \\
3 & 2.983 & $1.781-4.997$ & $<0.0001$ \\
4 & 2.922 & $1.625-5.252$ & 0.0047 \\
5 & 1.835 & $1.078-3.124$ & 0.9761 \\
6 & 2.670 & $1.368-5.211$ & 0.0940 \\
7 & 1.676 & $1.006-2.793$ & 0.3316 \\
8 & 1.658 & $0.917-2.999$ & 0.5365 \\
9 & 1.548 & $0.870-2.754$ & 0.2687 \\
10 & 1.564 & $0.940-2.601$ & 0.0910 \\
11 & 1.762 & $1.053-2.947$ & 0.6623 \\
\hline LV ejection fraction & 0.920 & $0.912-0.928$ & $<0.0001$ \\
\hline
\end{tabular}

3 or more inotropic

agents at the time of

incision

Yes versus no

4.859

$3.396-6.952$

$<0.0001$

CPR: cardiopulmonary resuscitation; ABO: blood groups; UNOS: United Network of Organ Sharing; LV: left ventricle.

not find a correlation between CPR donor heart utilization and regional transplantation volumes or wait times. This information is unique to our study and has not been studied

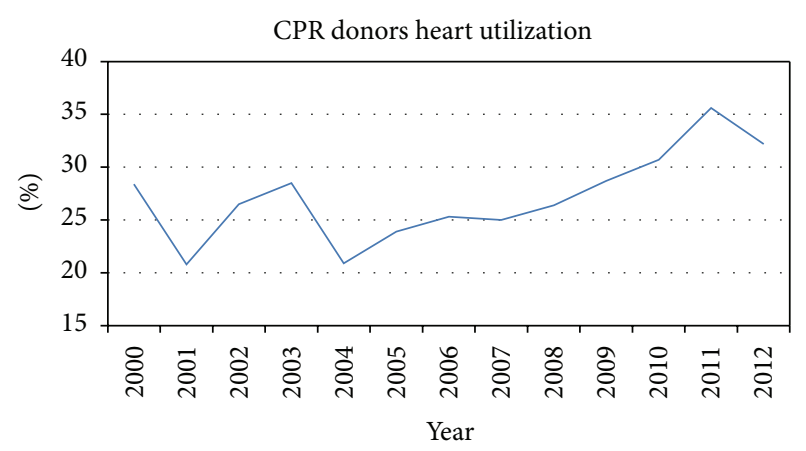

FIGURE 3: CPR donor heart procurement for transplantation between 2000 and 2012 .

and reported before. We noted a small but encouraging difference in utilization of CPR donor hearts over time, from $28.4 \%$ in 2000 to $32.2 \%$ in 2012 (Figure 3).

With the implementation of onsite CPR protocols, including both the advanced training of emergency medical response personnel and the increased availability of automated electrical defibrillators to the lay public, an increasing number of cardiopulmonary arrest victims are receiving CPR onsite and are being brought to the hospitals for continued resuscitation [12]. Unfortunately, a large percentage of these patients, as high as $70 \%$ arriving to hospitals after an onsite resuscitation with return of spontaneous circulation, are sustaining severe permanent brain damage $[13,14]$ and are then evaluated for organ donation. Over the past decade, the number of CPR donors have increased from $4.8 \%$ of the total donor pool in the year 2000 to $9.1 \%$ in the year 2012 (Figure 1); a $90 \%$ increase over time [2]. However, concerns remain regarding the optimal posttransplantation outcomes from CPR donors $[9,15]$, despite multiple studies demonstrating noninferior outcomes after heart transplantation from such donors $[6,16]$. Presently, only one-third of the potential CPR donor hearts are procured for heart transplantation [2]. However, there has been an upward trend in accepting hearts from these donors (Figure 3).

Donor factors associated with suboptimal posttransplantation outcomes have been studied extensively. Older donor age $[5,10]$, hypertension, low EF [10, 17], diabetes [11, 17], excess alcohol intake [18], and cigarette smoking [19] are among the well-established risk factors. In our study, presence of these factors among the CPR donors was associated with less procurement rates of hearts for transplantation, correlating with the published literature.

Donor gender significantly influences the heart utilization rate. In our study, we noted male CPR donor heart procurement of $34 \%$ as compared to female donor heart procurement of only $23 \%$; this, too, is consistent with most of the other studies. In a large study of over 1800 organ donors, $72 \%$ of male donor organs were utilized for transplantation as compared to $28 \%$ of female donor organs [10]. The male donor gender preference is primarily influenced by the larger male donor body size that matches well for most male and for all female recipients. Additionally, heart transplantation from female donors has been associated with less than optimal 
posttransplantation outcomes, both early and late, especially if the recipients were male [20].

An organ donor's cause of death has been known to influence posttransplantation outcomes. In particular, death resulting from cerebrovascular accidents and intracranial bleeding is associated with poor posttransplantation outcomes $[10,21]$. This explains the low percentage utilization of hearts from the CPR donors with strokes in our study. The likely explanation for this observation is that perhaps those donors who develop massive strokes tend to be relatively older and carry significant cardiovascular risk factors, such as hypertension and diabetes. Both of these factors are associated with inferior posttransplantation outcomes.

Studies on mechanism of injury leading to brain death show that this also influences donor organ utilization. Trauma victims constitute $55 \%$ to $74 \%$ of organ donors [22]. In a study by Kutschmann et al., besides older donor age, nontraumatic causes of death were associated with decreased recipient postheart transplant survival with an odds ratio of 1.48 [23]. Similarly, another study demonstrated that traumatic head injury leading to brain death is associated with higher donor organ utilization: $61 \%$ versus $22 \%$ for donors without head injury as a cause of brain death [10]. Our study's findings also suggest similar CPR donor heart procurement from both traumatic injury donors and donors with head trauma leading to brain death.

Organ donation rates differ among ethnic groups and are influenced by cultural beliefs and practices. In our study, we observed different rates of CPR donor heart procurement for transplantation by ethnicity. Donor heart procurement for transplantation from Hispanics, African Americans, Caucasians, and Asian ethnic groups was 37\%, 33\%, 27\%, and $24 \%$, respectively. This disparity in donor heart selection was also noted in another study where proportionately more Hispanic (30\%) and fewer Asian donors (5\%) were selected for heart procurement for transplantation following brain death compared to other ethnicities studied [10]. In our study, higher prevalence of head trauma (35\%), male gender $(62 \%)$, and blood group O (60\%) was noted in Hispanic ethnicity heart donors compared to other heart donors; it is possible that these attributes in isolation or in combination have contributed to higher heart procurement from Hispanic ethnicity heart donors.

The most common blood group among US population is $\mathrm{O}(44 \%)$ followed by $\mathrm{A}(42 \%), \mathrm{B}(10 \%)$, and $\mathrm{AB}(4 \%)$. Since donors and recipients are matched primarily by blood groups, it is expected that donors with O blood group (socalled universal donors) would be utilized most frequently. Heart procurement for transplantation among CPR donors in our study is consistent with this expectation.

Transplantation volumes and duration of wait times on the transplantation list vary at different centers and depend on the prevalence of heart failure within the patient population, the number of competing programs in the region, and, most importantly, the surrounding population. Posttransplantation outcomes have been linked to a center's transplantation volume, with low volume centers showing inferior survival [24]. In a study by Hosenpud et al., the critical heart transplantation center volume was nine per year; below this, survival outcomes were considered poor [25]. In another study by Kilic et al., institutional factors were also identified as contributing to inferior posttransplantation outcomes [26]. There, the authors suggest that the "heart transplantation center's volume" should not be the sole indicator of center's quality in heart transplantation. When donor risk factors were taken into account and posttransplantation outcomes were compared to transplant center volumes, it was clear that the low-volume centers did poorly with high-risk donors [26]. The difference in posttransplantation outcome persisted even when both the recipient risk factors and the institutional factors were taken into account. When stratified by the recipient risk scores, the posttransplantation outcomes appear profoundly worse in transplantation of extended criteria donors to high-risk recipients. The authors concluded that consolidating the use of extended criteria donors to higher volume centers might be prudent in improving postheart transplantation outcomes in high-risk recipients. The findings of the Kilic et al. study should be interpreted with caution, as the donor variables studied were few and transplant volumes defined as "low" were below 14 heart transplants per year. To follow this recommendation, it would prohibit greater than $77 \%$ of heart transplantation centers in the US from using high-risk donors [19]. In the same study, extended criteria donor utilization varied by the UNOS regions, with Region 8 utilizing $11.4 \%$ of marginal donors compared to $24.7 \%$ in Region 9 [26]. In our study, the highest percentage utilization of CPR donor hearts was in Region 1 (37\%) and the lowest was in Region 3 (24\%). We believe the difference in our results stems from different definitions of donor heart utilization: high-risk versus CPR donors. Time on 1A status varied significantly among the 11 regions, from a median of 35 days in Region 5 to 90 days in Region 7. It is possible to assume that the regions with longer wait times would likely accept extended criteria donors more often, including those who received CPR. In our study, however, longer wait times in regions did not correlate with increased CPR donor heart utilization. Similarly, our findings on the CPR donor heart utilization by population per heart transplantation performed in the 11 regions did not support any correlation between population dense regions and CPR donor heart utilization.

\section{Study Limitations}

Limitations inherent to retrospective data analysis are applicable to our study. The primary data source for our study is a composite UNOS database from more than 150 US transplantation centers. Completeness of all data fields is wanted and is not always available in the database. However, its merit lies in the fact that large, pooled data from different transplant centers eliminate biases associated with individual center practices. Thus, certain generalizations can be made of the results with regard to practicality of its usage and reproducibility. Since our focus was on the characteristics of CPR donors only, this limited the study population to $11 \%$ of the total donor pool, thus eliminating a majority of donors from our analysis. Since CPR donors comprise 
a potential group of underutilized heart donors, we felt it is important that we focus on this small but important group of donor hearts. We used the large UNOS database to help define donor characteristics that were associated with heart procurement for transplantation with the hope that in future at least CPR donor hearts with similar characteristics would be utilized more frequently for transplantations. Accepting a heart from an extended criteria donor also depends on the medical condition and acuity of illness of the recipient. The present study has not taken the recipient factors into consideration when defining CPR donor heart utilization. In our previous study of CPR heart donors, we noticed that more CPR donors were matched to recipients with high acuity of illness at transplantation, such as those with status 1A listing, those that were admitted to the hospital at transplantation, and those that were supported in an intensive care unit on life-sustaining support [6]. Despite the higher acuity of illness in the recipients, the use of selected CPR donor hearts did not negatively influence the intermediate or long-term outcomes of heart transplantation. Transplantation center practices do change over time with changes in leadership, philosophy, and patient population. We did not study the temporal changes in CPR donor heart utilization over time for each transplant region, or for each transplant center. With over 150 transplantation centers in 11 UNOS regions, we feel that 15 years of temporal data would be both cluttered in presentation and not very meaningful to the reader.

\section{Conclusions}

CPR donors are an expanding group of potential, yet presently underutilized, heart donors. With improvements in onsite resuscitative measures, more patients are regaining spontaneous circulation after CPR. However, if irreversible neurologic damage is sustained, these patients become eligible for organ donation. Though the clinical outcomes from accepting hearts from these donors are comparable, the overall utilization of these donor hearts is only $28.8 \%$. Donor characteristics such as younger age, male gender, absence of hypertension, smoking history, traumatic head injury, and shorter CPR duration are associated with higher heart procurement rates for transplantation. Regional factors do play a role in donor heart selection, but it does not seem to be associated with either recipient wait times or regional transplantation volumes. It is our hope that in the future CPR donor hearts with attributes associated with successful heart procurement for transplantation will not be passed up and that serious efforts will be made to accept these hearts, thus helping patients waiting desperately for transplantation.

\section{Conflict of Interests}

The authors declare that there is no conflict of interests regarding the publication of this paper.

\section{Acknowledgments}

The authors are indebted to the UNOS for providing the valuable data for analysis. The authors thank Mr. Adam Goodreau for his expert assistance in editing the paper.

\section{References}

[1] D. R. Wheeldon, C. D. O. Potter, A. Oduro, J. Wallwork, and S. R. Large, "Transforming the "unacceptable" donor: outcomes from the adoption of a standardized donor management technique," Journal of Heart and Lung Transplantation, vol. 14, no. 4, pp. 734-742, 1995.

[2] OPTN/SRTR 2011 Annual Data Report, HHS/ HRSA/HSB/ DOT, http://srtr.transplant.hrsa.gov/annual_reports/2011/flash/ 05 heart/index.html.

[3] A. K. Singhal, J. D. Abrams, J. Mohara et al., "Potential suitability for transplantation of hearts from human non-heart-beating donors: Data review from the Gift of Life Donor Program," Journal of Heart and Lung Transplantation, vol. 24, no. 10, pp. 1657-1664, 2005.

[4] M. Cypel and S. Keshavjee, "Extracorporeal lung perfusion," Current Opinion in Organ Transplantation, vol. 16, no. 5, pp. 469-475, 2011.

[5] M. D. Samsky, C. B. Patel, A. Owen et al., "Ten-year experience with extended criteria cardiac transplantation," Circulation Heart Failure, vol. 6, no. 6, pp. 1230-1238, 2013.

[6] M. A. Quader, L. G. Wolfe, and V. Kasirajan, "Heart transplantation outcomes from cardiac arrest-resuscitated donors," The Journal of Heart and Lung Transplantation, vol. 32, no. 11, pp. 1090-1095, 2013.

[7] C. S. Matsumoto, S. S. Kaufman, R. Girlanda et al., "Utilization of donors who have suffered cardiopulmonary arrest and resuscitation in intestinal transplantation," Transplantation, vol. 86, no. 7, pp. 941-946, 2008.

[8] K. Pilarczyk, B. R. Osswald, N. Pizanis et al., "Use of donors who have suffered cardiopulmonary arrest and resuscitation in lung transplantation," European Journal of Cardio-Thoracic Surgery, vol. 39, no. 3, pp. 342-347, 2011.

[9] E. Keitel, T. Michelon, A. F. dos Santos et al., "Renal transplants using expanded cadaver donor criteria," Annals of Transplantation, vol. 9, no. 2, pp. 23-24, 2004.

[10] K. K. Khush, R. Menza, J. Nguyen, J. G. Zaroff, and B. A. Goldstein, "Donor predictors of allograft use and recipient outcomes after heart transplantation," Circulation: Heart Failure, vol. 6, no. 2, pp. 300-309, 2013.

[11] A. O. Ojo, A. B. Leichtman, J. D. Punch et al., "Impact of preexisting donor hypertension and diabetes mellitus on cadaveric renal transplant outcomes," American Journal of Kidney Diseases, vol. 36, no. 1, pp. 153-159, 2000.

[12] J. Hollenberg, L. Svensson, and M. Rosenqvist, "Out-of-hospital cardiac arrest: 10 years of progress in research and treatment," Journal of Internal Medicine, vol. 273, no. 6, pp. 572-583, 2013.

[13] C. M. Booth, R. H. Boone, G. Tomlinson, and A. S. Detsky, "Is this patient dead, vegetative, or severely neurologically impaired? Assessing outcome for comatose survivors of cardiac arrest," Journal of the American Medical Association, vol. 291, no. 7, pp. 870-879, 2004.

[14] J. Berdowski, R. A. Berg, J. G. P. Tijssen, and R. W. Koster, "Global incidences of out-of-hospital cardiac arrest and survival rates: systematic review of 67 prospective studies," Resuscitation, vol. 81, no. 11, pp. 1479-1487, 2010. 
[15] D. J. Wilson, A. Fisher, K. Das et al., "Donors with cardiac arrest: improved organ recovery but no preconditioning benefit in liver allografts," Transplantation, vol. 75, no. 10, pp. 1683-1687, 2003.

[16] A. A. Ali, E. Lim, M. Thanikachalam et al., "Cardiac arrest in the organ donor does not negatively influence recipient survival after heart transplantation.," European Journal of CardioThoracic Surgery, vol. 31, no. 5, pp. 929-933, 2007.

[17] A. O. Ojo, J. A. Hanson, H. Meier-Kriesche et al., "Survival in recipients of marginal cadaveric donor kidneys compared with other recipients and wait-listed transplant candidates," Journal of the American Society of Nephrology, vol. 12, no. 3, pp. 589-597, 2001.

[18] D. J. Verran, T. Kusyk, D. Painter et al., "Clinical experience gained from the use of 120 steatotic donor livers for orthotopic liver transplantation," Liver Transplantation, vol. 9, no. 5, pp. 500-505, 2003.

[19] M. Berman, K. Goldsmith, D. Jenkins et al., "Comparison of outcomes from smoking and nonsmoking donors: thirteen-year experience," The Annals of Thoracic Surgery, vol. 90, no. 6, pp. 1786-1792, 2010.

[20] L. H. Lund, L. B. Edwards, A. Y. Kucheryavaya et al., “The registry of the international society for heart and lung transplantation: thirtieth official adult heart transplant report-2013; focus theme: age," The Journal of Heart and Lung Transplantation, vol. 32, no. 10, pp. 951-964, 2013.

[21] S. K. Akkina, S. K. Asrani, Y. Peng, P. Stock, W. R. Kim, and A. K. Israni, "Development of organ-specific donor risk indices," Liver Transplantation, vol. 18, no. 4, pp. 395-404, 2012.

[22] R. C. Mackersie, O. L. Bronsther, and S. R. Shackford, "Organ procurement in patients with fatal head injuries: the fate of the potential donor," Annals of Surgery, vol. 213, no. 2, pp. 143-150, 1991.

[23] M. Kutschmann, C. L. Fischer-Fröhlich, I. Schmidtmann et al., "The joint impact of donor and recipient parameters on the outcome of heart transplantation in Germany after graft allocation," Transplant International, vol. 27, no. 2, pp. 152-161, 2014.

[24] G. L. Laffel, A. I. Barnett, S. Finkelstein, and M. P. Kaye, “The relation between experience and outcome in heart transplantation," The New England Journal of Medicine, vol. 327, no. 17, pp. 1220-1225, 1992.

[25] J. D. Hosenpud, T. J. Breen, E. B. Edwards, O. P. Daily, and L. G. Hunsicker, "The effect of transplant center volume on cardiac transplant outcome: a report of the United Network for Organ Sharing Scientific Registry," Journal of the American Medical Association, vol. 271, no. 23, pp. 1844-1849, 1994.

[26] A. Kilic, E. S. Weiss, D. D. Yuh et al., "Institutional factors beyond procedural volume significantly impact center variability in outcomes after orthotopic heart transplantation," Annals of Surgery, vol. 256, no. 4, pp. 616-623, 2012. 


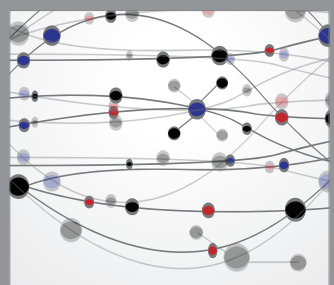

The Scientific World Journal
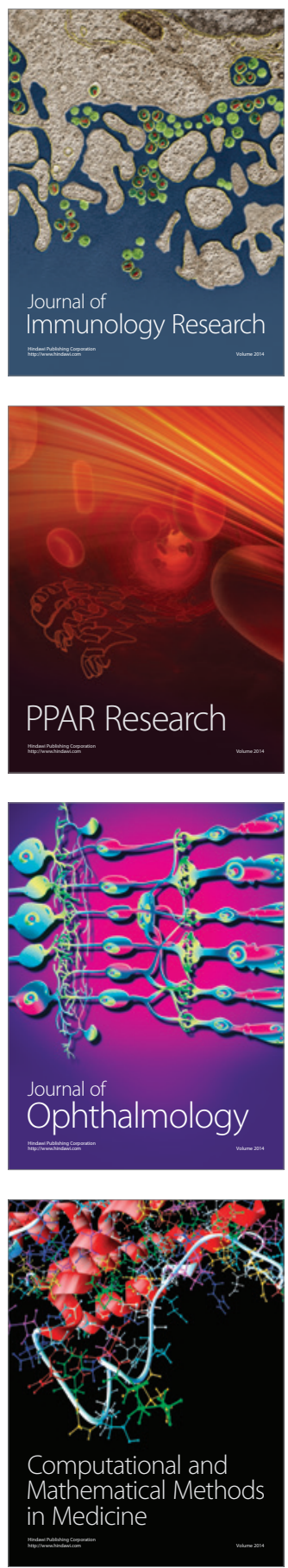

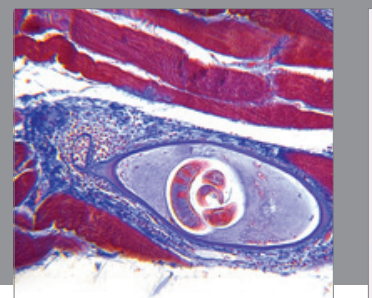

Gastroenterology

Research and Practice
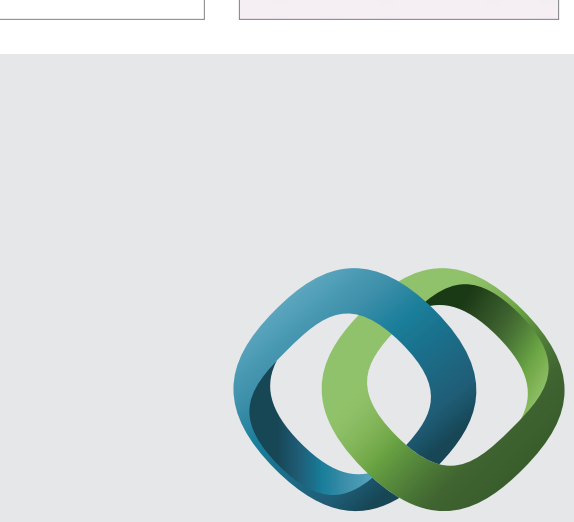

\section{Hindawi}

Submit your manuscripts at

http://www.hindawi.com
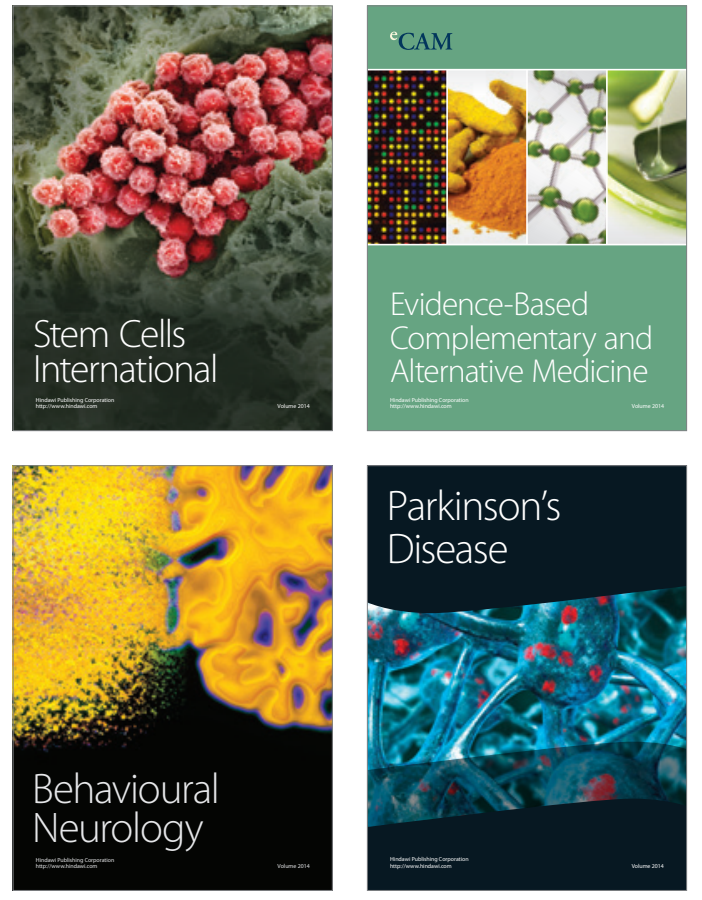
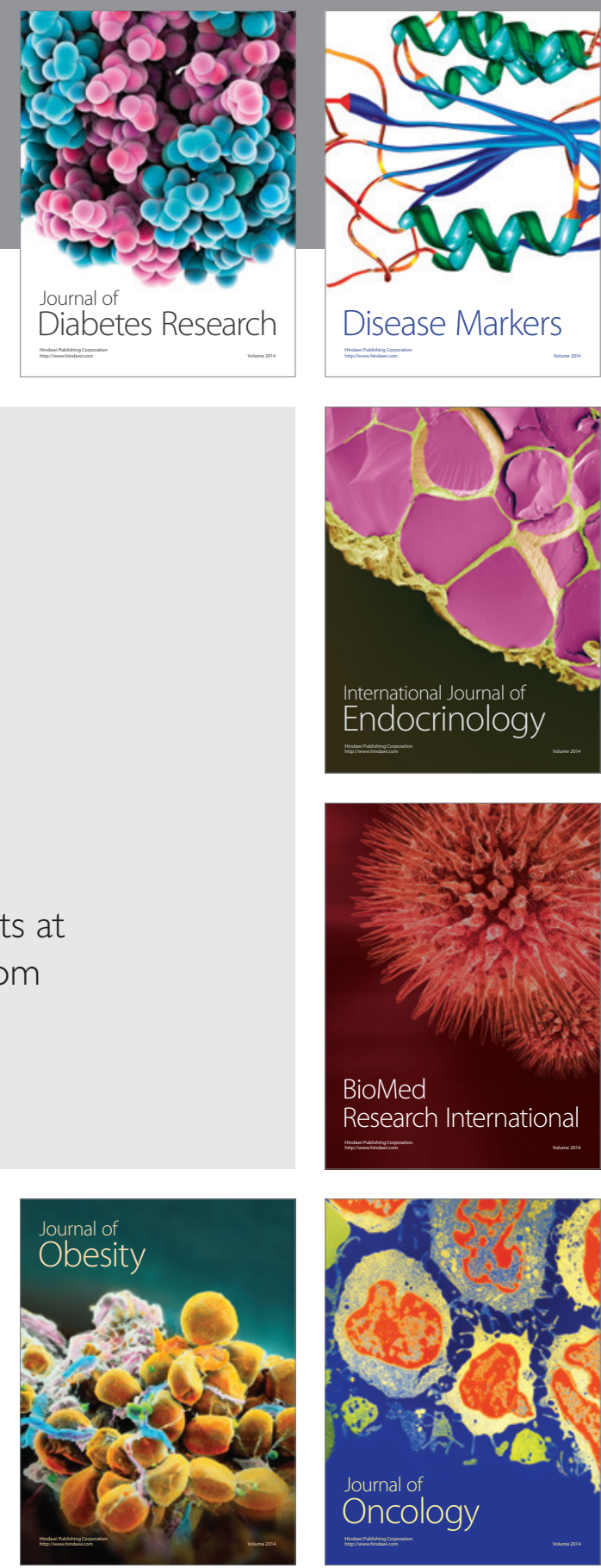

Disease Markers
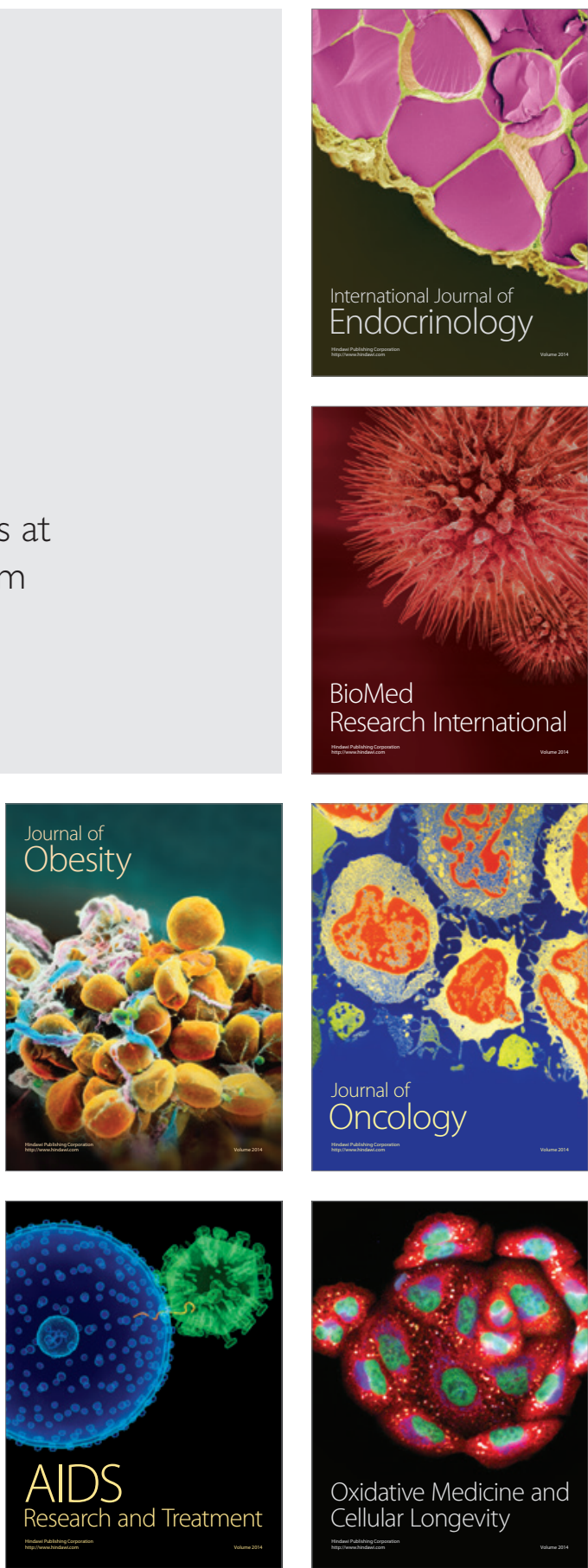